\title{
An antiviral trap made of protein nanofibrils and iron oxyhydroxide nanoparticles
}

\author{
Archana Palika', Antonius Armanious ${ }^{1}{ }^{1}$, Akram Rahimi 1,2, Chiara Medaglia33, Matteo Gasbarri ${ }^{\circ} 4$, \\ Stephan Handschin', Antonella Rossi ${ }^{5,6}$, Marie O. Pohl', Idoia Busnadiego7, Christian Gübeli', \\ Ravi B. Anjanappa ${ }^{8}$, Sreenath Bolisetty ${ }^{1,2}$, Mohammad Peydayesh ${ }^{\circ}$, Silke Stertz ${ }^{7}$, Benjamin G. Hale ${ }^{7}{ }^{7}$, \\ Caroline Tapparel ${ }^{3}$, Francesco Stellacci ${ }^{4}{ }^{4,9}$ and Raffaele Mezzenga ${ }^{1,6}{ }^{1,}$
}

\begin{abstract}
Minimizing the spread of viruses in the environment is the first defence line when fighting outbreaks and pandemics, but the current COVID-19 pandemic demonstrates how difficult this is on a global scale, particularly in a sustainable and environmentally friendly way. Here we introduce and develop a sustainable and biodegradable antiviral filtration membrane composed of amyloid nanofibrils made from food-grade milk proteins and iron oxyhydroxide nanoparticles synthesized in situ from iron salts by simple pH tuning. Thus, all the membrane components are made of environmentally friendly, non-toxic and widely available materials. The membrane has outstanding efficacy against a broad range of viruses, which include enveloped, non-enveloped, airborne and waterborne viruses, such as SARS-CoV-2, H1N1 (the influenza A virus strain responsible for the swine flu pandemic in 2009) and enterovirus 71 (a non-enveloped virus resistant to harsh conditions, such as highly acidic pH), which highlights a possible role in fighting the current and future viral outbreaks and pandemics.
\end{abstract}

T he current ongoing COVID-19 pandemic illustrates the importance of valid therapeutic tools ${ }^{1}$, but also the notable lack of technologies capable of fighting the spread of viruses in the environment. Many viruses diffuse and transmit in the environment in water, some in their bulk form (waterborne viruses) ${ }^{2-4}$ and others in small droplets suspended in the air (airborne viruses) ( $^{5-9}$. A key defence strategy against infectious diseases is always the prevention of pathogen transmission from an infected person to an uninfected one. This is achieved by using masks, gloves, physical barriers and disinfection, which introduces other challenges on a global scale, such as achieving the goal in a fully sustainable and environmentally friendly way ${ }^{10-12}$.

In particular, the COVID-19 pandemic revealed the paradox that, although both technological and scientific knowledge are available to develop a vaccine within the record time of less than a year ${ }^{13,14}$, there is still a lack of preparedness to fight the rapid spread of new viruses until such vaccines are developed and a substantial portion of the population is vaccinated ${ }^{15}$. Without the appropriate readiness, viruses spread widely and rapidly and, eventually, new virus strains emerge via mutations ${ }^{16,17}$, which could potentially confer resistance to vaccines that target the original strain or increase the virulence of the virus, and possibly lead to an endless vicious cycle. Viruses can spread through many different routes, but mostly through fomites ${ }^{18}$, small water droplets ${ }^{5-9}$ and bulk water bodies (which include wastewater) ${ }^{2-4}$. Proper hand hygiene serves as a very effective practice against the spread of infections through fomites ${ }^{19}$. In all the other cases, however, virus inactivation must be tackled in the hosting fluid, and the overarching strategy is then to target the virus in its surrounding aqueous environment, may this be in the form of microscopic suspended water droplets or bulk waters.
For airborne viruses, suitable face masks, although effective, pose the risk of further dissemination of the viruses when improperly handled $^{20}$ and/or disposed ${ }^{21,22}$. Additionally, the generated plastic waste eventually emerges as a parallel environmental problem, especially in times of pandemics ${ }^{10-12}$. For waterborne viruses, and despite decades-long technological developments, contaminated drinking water is still responsible for 500,000 annual deaths, of which more than half occur in children under five years of age ${ }^{23}$. Non-enveloped enteric viruses, such as enteroviruses, adenoviruses and rotaviruses, can cause gastrointestinal infections with diseases such as diarrhoea and dysentery. It is estimated that $\sim 40 \%$ of often-fatal childhood diarrhoea in developing countries is connected to viral agents ${ }^{24}$. Non-enveloped viruses can persist in water bodies for long periods of time ${ }^{25}$ and can resist even some of the harshest treatments ${ }^{26,27}$. This challenge is not only exclusive to unfavoured communities, but extends also to countries with state-of-the-art water and wastewater treatment facilities ${ }^{2}$. Furthermore, even enveloped viruses, such as influenza viruses and coronaviruses, which were often regarded as unstable in water environments, have now been shown to remain highly infective for long times in bulk water bodies ${ }^{28,29}$ : SARS-CoV-2, for example, can retain its infectivity for longer than seven days in tap water and wastewater at room temperature ${ }^{30}$.

Therefore, the development of efficient barriers against the spread of viruses via diffusing environmental fluids becomes crucial if global contamination is to be prevented. In spite of decades of scientific and technological development, no existing technology can universally eliminate viruses from water, unless it is extremely energy intensive (for example, reverse osmosis) ${ }^{31}$ or starts to pose the risk of toxicity towards humans and the environment (for example, silver-based technologies) ${ }^{32,33}$. All these limitations

'Department of Health Sciences and Technology, ETH Zurich, Zurich, Switzerland. ${ }^{2}$ BluAct Technologies GmbH, Zurich, Switzerland. ${ }^{3}$ Department of Microbiology and Molecular Medicine, University of Geneva, Geneva, Switzerland. ${ }^{4}$ Institute of Materials, Ecole Polytechnique Fédérale de Lausanne (EPFL), Lausanne, Switzerland. ${ }^{5}$ Department of Chemical and Geological Science, University of Cagliari, Cagliari, Italy. ${ }^{6}$ Department of Materials, ETH Zurich, Zurich, Switzerland. ${ }^{7}$ Institute of Medical Virology, University of Zurich, Zurich, Switzerland. ${ }^{8}$ Department of Biology, ETH Zurich, Zurich, Switzerland.

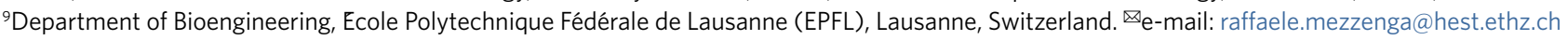


a

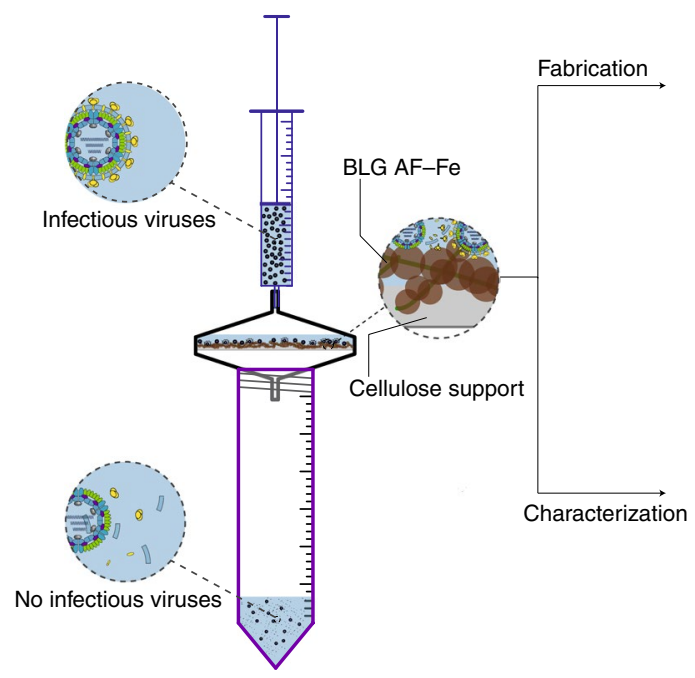

b

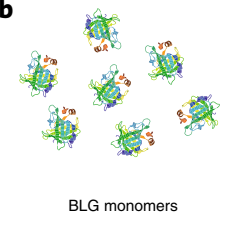

c
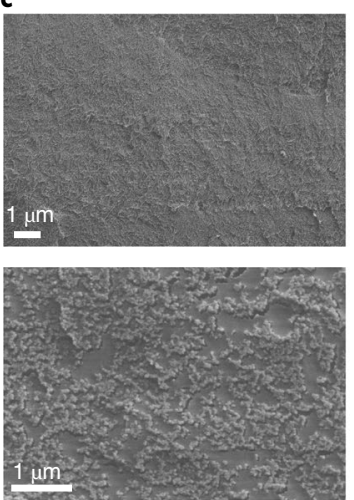
$5 \mathrm{~h}$
$\stackrel{\mathrm{pH} 2,90^{\circ} \mathrm{C}}{\longrightarrow}$

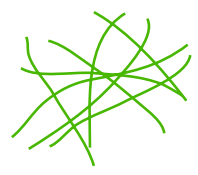

BLG AF

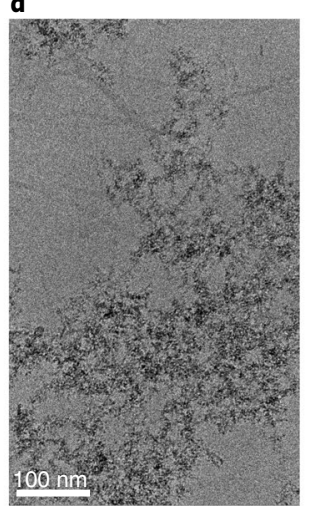

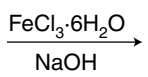

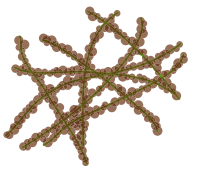

BLG AF-Fe

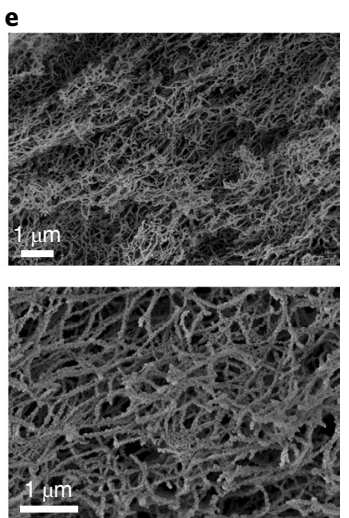

Fig. 1 | Schematic, fabrication and characterization of BLG AF-Fe membranes. $\mathbf{a}$, A schematic showing the filtration set-up. $\mathbf{b}$, A schematic showing the fabrication processes of the BLG AF-Fe membranes. c, Cryo-SEM micrographs of freeze-fractured and freeze-etched hydrated BLG AF-Fe hybrids at two magnifications. d, Cryo-TEM image of the BLG AF-Fe hybrids; the BLG AF-Fe hybrids were prepared using a fivefold lower Fe concentration (10 mgFe ml-1) than the concentration normally used to prepare the membranes to enable the visualization of single Fe NPs. e, SEM micrographs of a chemically fixed and critical point-dried sample of the filter bulk material at two magnifications. Representations of the virions in $\mathbf{a}$ are reproduced from pictures on ViralZone ${ }^{51}$. The cartoon structure of the BLG monomers in $\mathbf{b}$ is based on the crystallographic structures $5105^{52}$ obtained from the Protein Data Bank ${ }^{53}$ (http://www. rcsb.org/, accessed October 2020).

render the existing technologies obsolete facing global challenges such as pandemics.

To address such a challenge, we developed an antiviral membrane trap composed of amyloid nanofibrils obtained from a food-grade milk protein, $\beta$-lactoglobulin (BLG), modified in situ with iron oxyhydroxide nanoparticles (NPs) (Fig. 1a). The conversion of BLG monomers into a network of amyloid fibrils (AFs) is a straightforward process achieved by simply lowering the $\mathrm{pH}$ to 2.0 with simultaneous heating up to $90^{\circ} \mathrm{C}$; afterwards, the iron oxyhydroxide NPs are precipitated directly on the formed network of AFs by raising the $\mathrm{pH}$ in the presence of $\mathrm{FeCl}_{3} \cdot 6 \mathrm{H}_{2} \mathrm{O}$. Figure $1 \mathrm{~b}$ shows a schematic of the process; the detailed chemical analysis and composition of the iron NPs as studied by X-ray photoelectron spectroscopy (XPS) reveals the presence of both iron(II) and iron(III) oxide and iron chloride species that coexist with the majority of iron oxyhydroxide NPs (Supplementary Fig. 1). By depositing the produced material on a porous solid support, we obtained a filtration membrane composed of an intricate network of BLG AFs decorated by Fe oxyhydroxide NPs a few nanometres in size (in what follows, the hybrid membrane is simply referred to as BLG AF-Fe and the decorating iron nanoparticles as Fe NPs; BLG AF-Fe hybrids refer instead to the solution precursor). The resulting membrane is thus composed of food-grade components and shows no toxicity on experimentally treated cell lines, as demonstrated by cytotoxicity tests (Supplementary Fig. 2). The general structure of the membrane can be clearly observed by cryogenic scanning electron microscopy (cryo-SEM) (Fig. 1c) on a fracture plane through the inner structure of the fully hydrated filter: controlled etching lowers the level of water and enhances the visibility of the BLG AF-Fe hybrids. Alternatively, Fe NPs that decorate the AF can be visualized by cryogenic transmission electron microscopy (cryo-TEM) (Fig. 1d) on samples prepared using a fivefold lower Fe concentration to allow the resolution of single NPs. Finally, scanning electron microscopy (SEM) micrographs (Fig. 1e) of fully dried membranes reveal, in high-contrast images, the dense packing of the Fe NPs on the surface of AFs. It is important to mention that the observed difference in the Fe NP density between the cryo-SEM (Fig. 1c) and the SEM (Fig. 1e) micrographs is due to the shrinkage of the sample during preparation and drying.

\section{Performance of membranes on enveloped viruses}

We first tested this filtration membrane trap by filtering water that contained three different types of enveloped viruses: (1) $\Phi 6$, an enveloped bacteriophage that infects Pseudomonas syringae bacteria and is often used as a surrogate of human enveloped viruses ${ }^{34}$, (2) H1N1, an influenza A virus strain responsible for the swine flu pandemic in $2009^{35}$ and (3) SARS-CoV-2, the coronavirus strain responsible for the ongoing COVID-19 pandemic. The membrane showed an efficiency of more than six orders of magnitude reduction in infectivity for all three viruses (Fig. 2). The infectious virus concentrations went from $\sim 10^{6} \mathrm{PFU} \mathrm{ml}^{-1}$ before filtration to below the detection limit after filtration for all three viruses. No remarkable effect on the infectivity of the viruses was observed when filtering the viruses through the cellulose support or the BLG AF alone, which suggests a unique synergetic effect of the BLG AF-Fe membranes. The filter has a capacity of $\sim 7 \times 10^{3}$ PFU mg BLG AF-Fe $^{-1}$ (Supplementary Fig. 4), as determined both by repeated cycles of filtration and by varying the total volume of the BLG AF-Fe hybrid solution used to prepare the final membrane.

By quantifying the viral genomes, which indicate the total number of viruses (both infectious and non-infectious), before and after filtration (Fig. 2), we observed that most of the viruses were retained on the membrane material. Still, for H1N1 and SARS-CoV-2, a detectable amount of genomes passed through the filter. It is, however, important to reiterate that the infective viruses in the filtrate were below the detection limit for both viruses. Even in the conservative assumption that the concentration of the infective virus in the filtrate is equal to the detection limit, we still observed a remarkable decrease in the ratio of infective to total viruses after filtration 


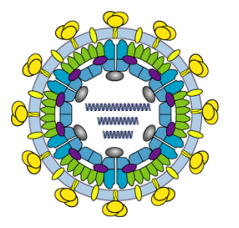

$\Phi 6$

$85 \mathrm{~nm}$ b

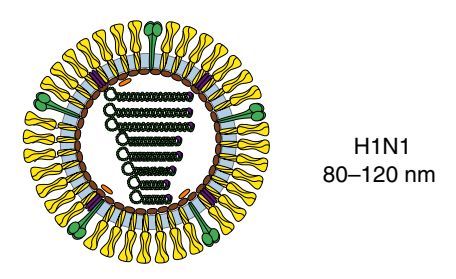

C

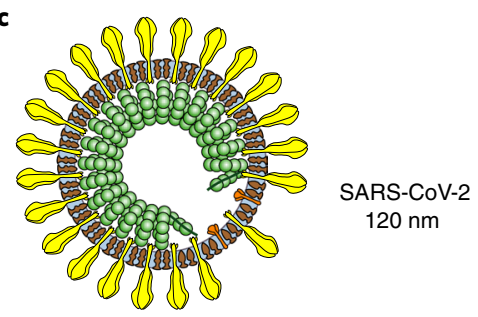

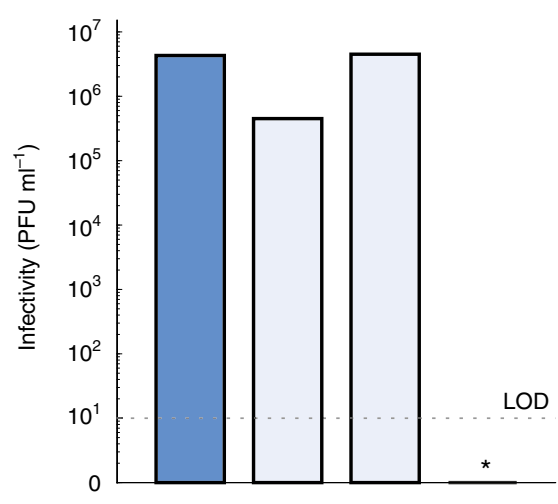

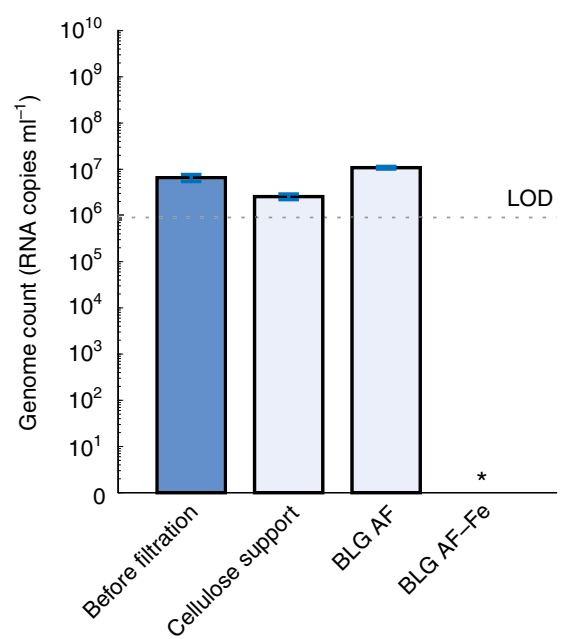

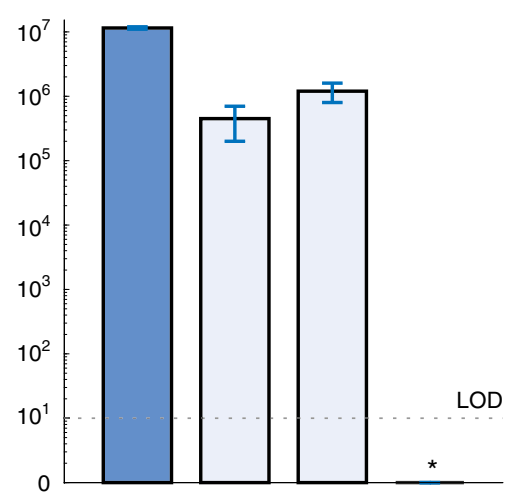
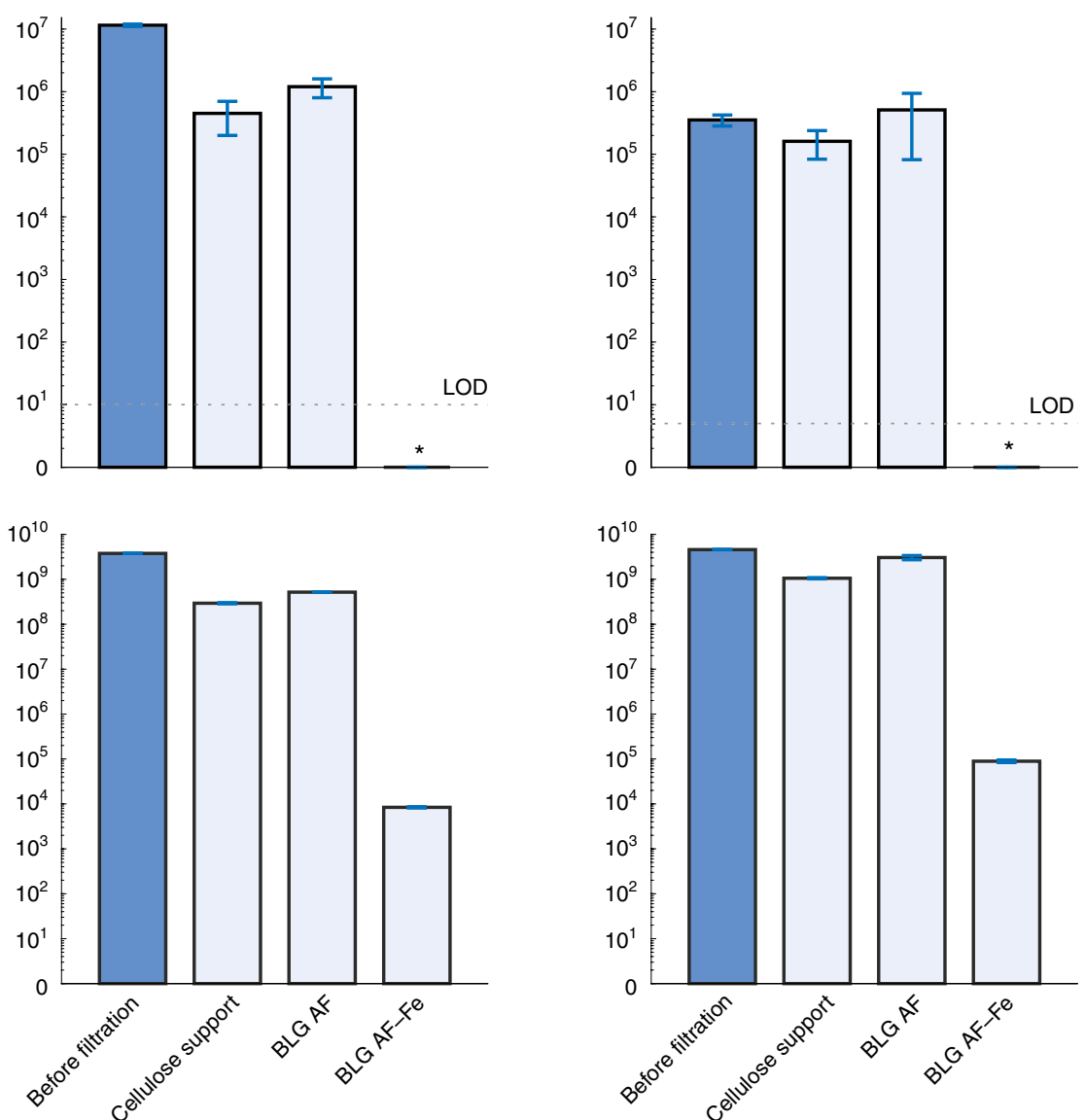

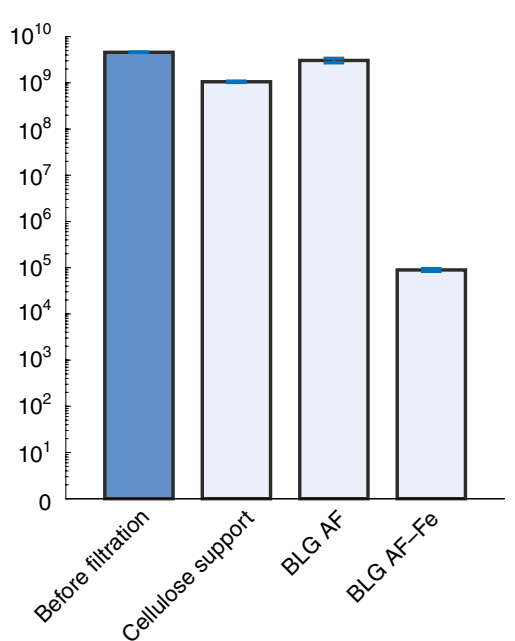

Fig. 2 | Elimination of infectious enveloped viruses for water filtered through BLG AF-Fe membranes. a-c, Complete elimination of infectious viruses and the corresponding reduction in the genome count for $\Phi 6$ (a), H1N1 (b) and SARS-CoV-2 (c) when filtered through BLG AF-Fe membranes (blue, before filtration; grey, after filtration). A limited or no elimination was observed when filtering the same viruses through the cellulose support or the BLG AFs alone. The lower value of the genome count of $\Phi 6$ than that of the other two viruses is probably due to both a higher ratio of infectious viruses to genome count than those of the other two viruses and also the low efficiency of the genome extraction from these phages (Supplementary Table 2). $\Phi 6$ infectivity represents the plaque count from one plate of a series of dilutions that consist of at least three plates. A replicate of the $\Phi 6$ filtration experiment for which the infectivity was calculated using three technical replicas is shown in Supplementary Fig. 3. The genome count for $\Phi 6$ represents the average of four technical replicas and the error bars represent the standard deviation (s.d.). The infectivity and genome count of H1N1 as well as the genome count of SARS-CoV-2 represent the average of two technical replicas and the error bars represent the range. The infectivity of SARS-CoV-2 represents the average of four technical replicas and the error bars represent the s.d. LOD, limit of detection; *below the LOD. Representations of the virions are reproduced from pictures on ViralZone ${ }^{51}$.

(Supplementary Fig. 5). These results suggest that the membrane not only retains the virus, but also strongly inactivates it.

Further assessment of the virucidal effect of the membrane was conducted by attempting to recover the $\Phi 6$ viruses retained on the membrane filter. This was done by incubating the membrane material used for $\Phi 6$ filtration in a beef buffer of $\mathrm{pH} 9.3$-beef buffer has often been used to recover viruses adsorbed to iron oxides ${ }^{36}$. Keeping in mind the challenges of an efficient recovery of adsorbed viruses and potential damage to the virus in that process, we still observed a pronounced decrease in the ratio of infective to total viruses from 0.05 for the filtered solution to as low as $8.8 \times 10^{-4}$ of the recovered viruses (Supplementary Fig. 6). This observation provides additional evidence that the viruses not only adsorb to the membrane but also are mostly inactivated.

\section{Inactivation mechanisms}

To further investigate the mechanism by which the BLG AF-Fe membrane eliminates viruses, we ran experiments in which the 
a

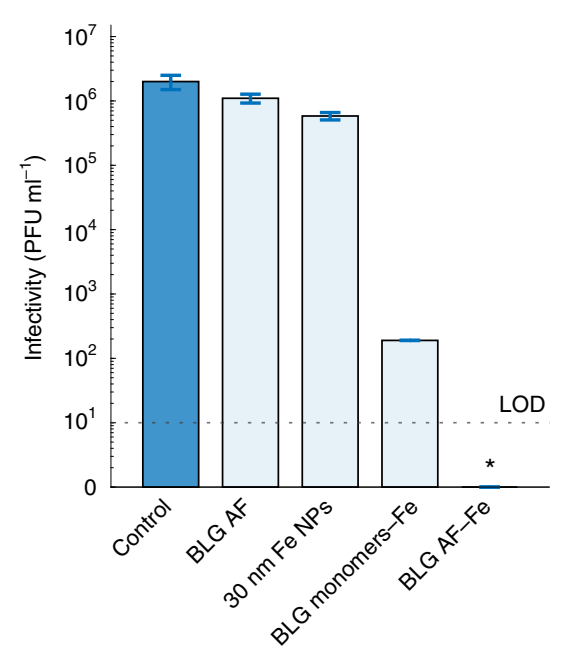

d

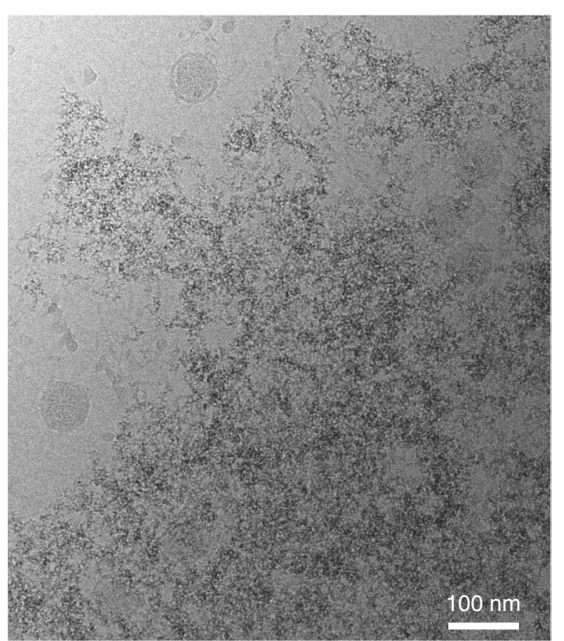

b
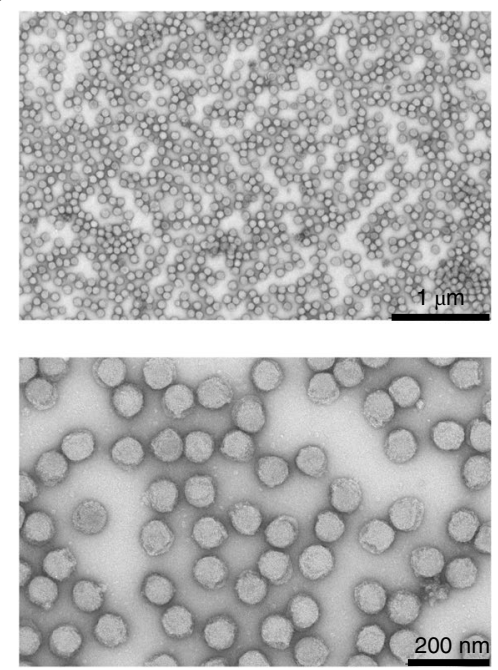

e

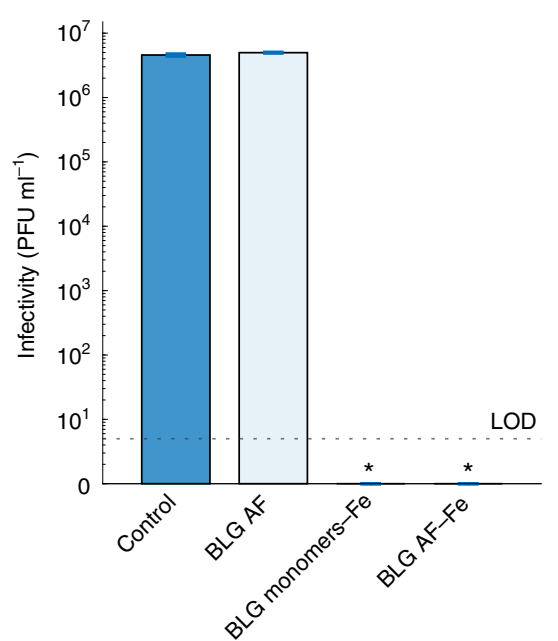

c
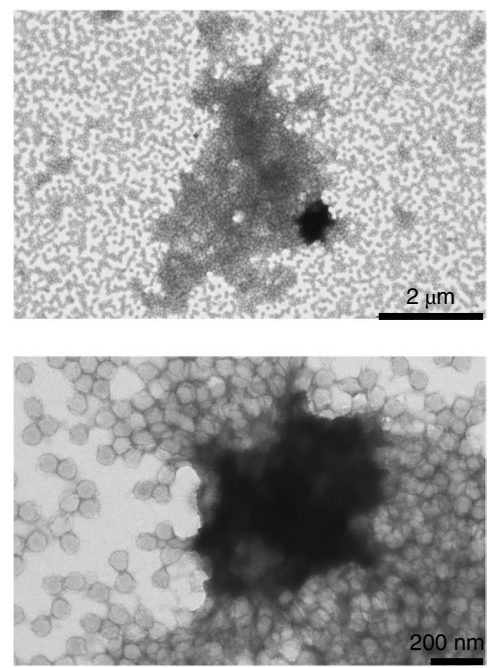

f

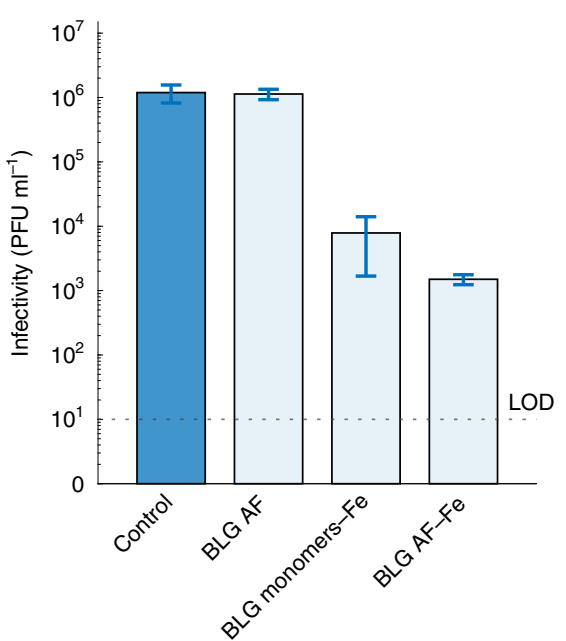

Fig. 3 | Mechanism of elimination of infective viruses by BLG AF-Fe hybrids. a, Infectivity of $\Phi 6$ after $1 \mathrm{~h}$ of incubation with BLG AF, $30 \mathrm{~nm}$ Fe NPs, BLG monomer-Fe and BLG AF-Fe. A control experiment was conducted by incubating the virus in PBS buffer without any additives. Complete elimination of infectious $\Phi 6$ is only achieved when incubated with BLG AF-Fe hybrids for 60 min; no or very limited elimination was observed when the viruses were incubated with BLG AF or 30 nm Fe NPs alone; elimination became substantial, yet partial, when the virus was incubated in BLG monomer-Fe. The plotted data are the average of three technical replicas and the error bars represent the s.d., except for the BLG monomer-Fe data, which are the average of two technical replicas and the error bars represent the range. b, TEM micrographs of negative stained $\Phi 6$ showing intact phages. $\mathbf{c}$, TEM micrographs of negative stained $\Phi 6$ incubated with BLG AF-Fe hybrids. d, Cryo-TEM micrograph of $\Phi 6$ incubated with BLG AF-Fe hybrids. Of the eight viruses in the field of view, two are not associated with BLG AF-Fe (left side) and six are associated (on the top right and lower end of the image) with BLG AF-Fe. No clear signs of structural damage to the viruses can be seen. e,f, Infectivity of H1N1 (e) and SARS-CoV-2 (f) after 1h of incubation with BLG AF, BLG monomer-Fe and BLG AF-Fe. A control experiment was conducted by incubating the virus in PBS buffer without any additives. Complete-to-partial elimination of infectious H1N1 and SARS-CoV-2 occurred when incubated with BLG AF-Fe hybrids. No elimination was observed when the viruses were incubated with BLG AF alone. A substantial elimination was observed when the viruses were incubated with BLG monomer-Fe. H1N1 data represent the average of two technical replicas and the error bars represent the range. SARS-CoV-2 data are the average of three technical replicas with error bars that represent the s.d. ${ }^{\star}$ Below the LOD.

viruses were incubated with a suspension of BLG AF-Fe hybrids in PBS buffer for one hour followed by assessing the infectivity of the viruses. No infective $\Phi 6$ viruses were detected in the solution at the end of the incubation time (Fig. 3a). These results show that virus elimination during filtration cannot be attributed to simple retention on the filter material due to small pore sizes, that is, by size exclusion: indeed, no change in the $\Phi 6$ infectivity was observed in control experiments when the viruses were incubated with BLG $\mathrm{AF}$ or Fe NPs alone. The reduction in infective viruses in solution started to become noticeable when the viruses were incubated with BLG monomer-Fe; still it was neither as effective nor as reproducible as incubation with BLG AF-Fe hybrids. These results were further supported by transmission electron microscopy (TEM) images of the $\Phi 6$ viruses alone (Fig. 3b), $\Phi 6$ incubated with BLG AF-Fe hybrids pre-adsorbed on carbon films (Fig. 3c) and $\Phi 6$ incubated with BLG AF-Fe hybrids imaged using cryo-TEM. Figure $3 \mathrm{~b}$ shows a high concentration of spherical and intact $\Phi 6$ virions. The top image of Fig. $3 \mathrm{c}$ shows that BLG AF-Fe is densely covered with $\Phi 6$ phages. It is important to note that the heavy metal staining used to produce contrast in the TEM image stabilizes single molecules, fibrils and virus particles adsorbed on the grid; bulky pieces of BLG AF-Fe are, however, too large and the stabilizing effect 
a

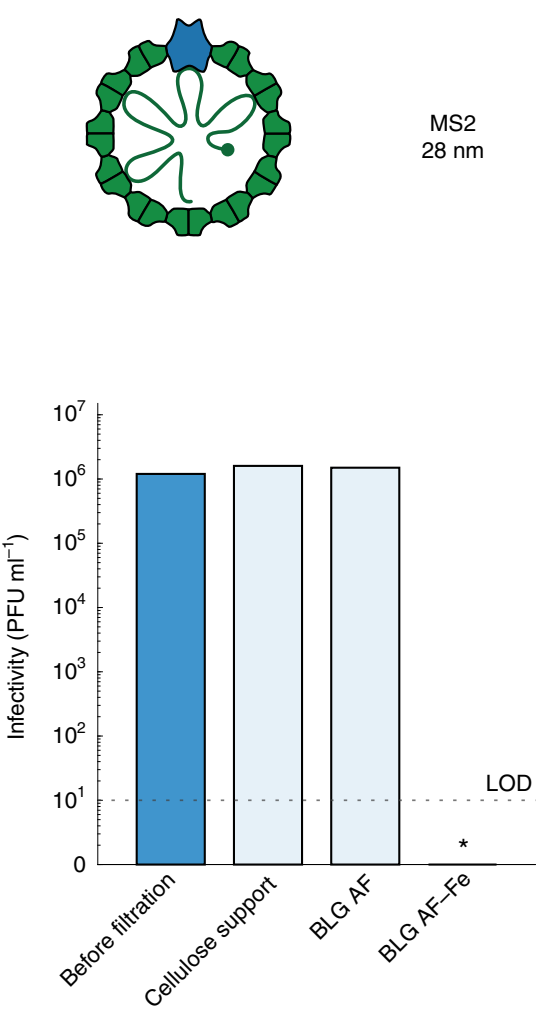

b

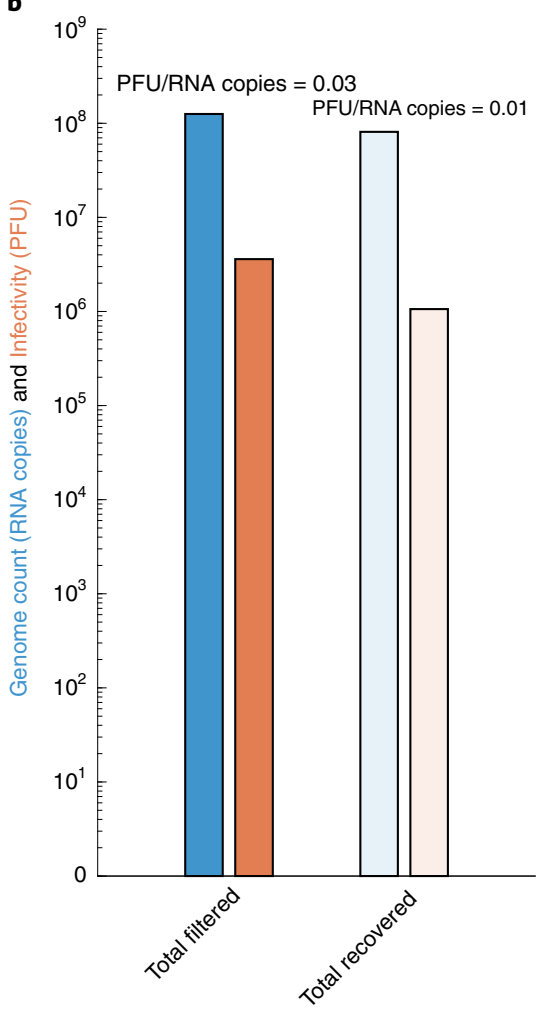

c
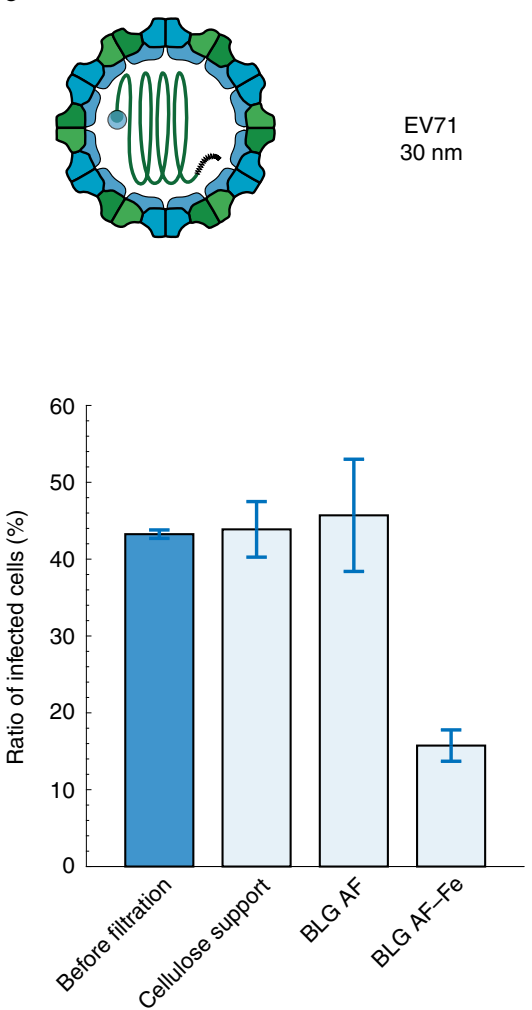

Fig. 4 | Complete and partial elimination of infectious non-enveloped viruses for water filtered through BLG AF-Fe membranes. a, Complete elimination of infectious MS2 viruses (the corresponding reduction in the genome count is shown in Supplementary Fig. 10) when filtered through BLG AF-Fe membranes (blue, before filtration; grey, after filtration). No detectable elimination was observed when filtering the same viruses through the cellulose support or the BLG AFs alone. The plotted infectivity represents the plaque count from one plate of a series of dilutions that consist of at least three

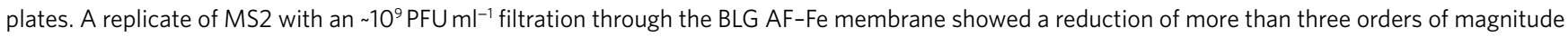
(data not shown). b. Genome count and infectivity of the total amount of MS2 viruses filtered and recovered: total genome count filtered = volume of filtered solution $(\mathrm{ml}) \times$ genome count before filtration (RNA copies $\mathrm{ml}^{-1}$ ); total infectious viruses filtered $=$ volume of filtered solution $(\mathrm{ml}) \times$ infectivity before filtration $\left(\mathrm{PFU} \mathrm{ml^{-1 }}\right)$; total genome count recovered $=$ volume of filtered solution $(\mathrm{ml}) \times$ genome count after filtration $\left(\right.$ RNA copies $\left.\mathrm{ml}^{-1}\right)+$ volume of beef buffer $(\mathrm{ml}) \times$ genome count in beef buffer after $1 \mathrm{~h}$ of incubation (RNA copies $\mathrm{ml}^{-1}$ ); total infectious viruses recovered $=$ volume of filtered solution $(\mathrm{ml}) \times$ infectivity after filtration (PFU ml-1) + volume of beef buffer $(\mathrm{ml}) \times$ infectivity in beef buffer after $1 \mathrm{~h}$ of incubation (PFU $\left.\mathrm{ml}^{-1}\right)$. Beef buffer $(\mathrm{pH} 9.3$ ) was used to desorb the viruses from the BLG AF-Fe. The genome count for MS2 represents the average of four technical replicas. $\mathbf{c}$, Substantial elimination of infectious enterovirus (EV71) viruses when filtered through BLG AF-Fe membranes. No detectable elimination was observed when the same viruses were filtered through the cellulose support or the BLG AFs alone. The results for EV71 represent the average of two technical replicas and the error bars represent the range. *Below the LOD. Representations of virions are reproduced from pictures on ViralZone ${ }^{51}$.

of the stain is not sufficient due to their spatial hydrogel nature and thus such staining cannot stop them from shrinking during the air-drying procedure after staining. The viruses attached at the surface of BLG AF-Fe hybrids (Fig. 3c) appear to be elongated in a direction perpendicular to the surface, which suggests that such elongation was driven by the shrinkage of the membrane on drying and indicates a very strong interaction between the viruses and the BLG AF-Fe hybrids. A weak interaction would have probably resulted in the release of the viruses from the surface of BLG AFFe hybrids on washing and drying the samples. Such strong interactions could be sufficient to inactivate the viruses, as previously observed for $\Phi 6$ viruses that interact with montmorillonite clay minerals ${ }^{37}$. Additional experiments conducted under anoxic conditions showed that no oxygen-mediated inactivation took place (Supplementary Fig. 8), which supports again that the attachment of the viruses to the BLG AF-Fe is the main mechanism behind the virus elimination. Incubation experiments with H1N1 (Fig. 3e) and SARS-CoV-2 (Fig. 3f) showed comparable results to those of $\Phi 6$, which suggests a similar elimination mechanism for all three viruses.
These results together show that the Fe NPs combined with the AF features are the key element of the membrane: BLG AF alone did not show any virucidal effect, whereas BLG monomer-Fe had a noticeable, yet moderate, inactivating effect on the viruses compared with that of BLG AF-Fe hybrids. Previous studies suggested that strong attraction forces between positively charged iron hydroxides ${ }^{38}$ and negatively charged viruses ${ }^{39}$ can partially inactivate the virus via interactions with its capsid ${ }^{40-42}$. Our results, indeed, confirm that the Fe NPs, the BLG monomer-Fe and BLG AF-Fe are all positively charged at physiological $\mathrm{pH}$ values (Supplementary Fig. 9), whereas literature values show that $\mathrm{H} 1 \mathrm{~N} 1^{43}$ and the spike protein of SARS-CoV- $2^{44}$ are both negatively charged at physiological $\mathrm{pH}$ values. What is critically important, however, is the surface-to-volume ratio at which these surfaces become available: the $30 \mathrm{~nm}$ Fe NP control had no virucidal effect on $\Phi 6$, the BLG monomer Fe had a noticeable, partial effect and only BLG AF-Fe had an outstanding effect. The BLG AF provide an intricate network template that supports the formation of an Fe coating a few nanometres thick onto their elongated surface, that is, at a remarkably higher surface-to-volume ratio than that offered by the 

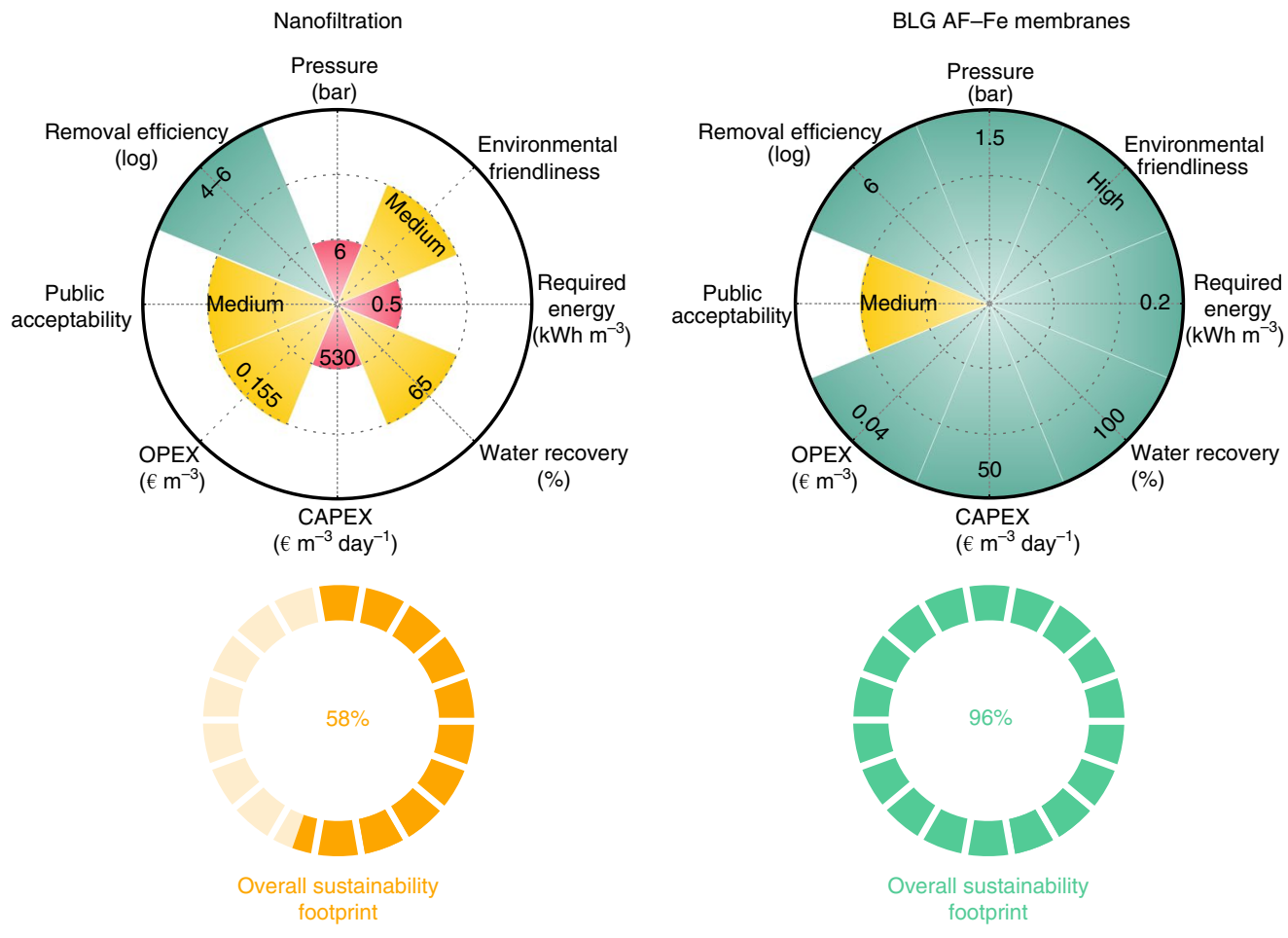

Fig. 5 | BLG AF-Fe membranes versus NF sustainability footprint. The factors considered are operating costs (OPEX), investment cost (CAPEX), energy consumption, water recovery, removal efficiency, pressure, public acceptability and environmental friendliness. The scores of technologies in each discriminant are shown by red (low performance), yellow (medium) and green (high performance). The overall sustainability footprint is obtained by a weighted averaging of the score in each discriminant (see the text for details). In this analysis, an industrial grade of BLG, that is, whey, is considered as the source for AF preparation.

spherical geometry of Fe NPs and BLG monomer-Fe NPs. We anticipate that further insights into virus inactivation mechanisms within the BLG AF-Fe membranes could be gained with techniques such as radioactive labelling of the different components of the viruses, genome-wide PCR analysis and/or a variety of mass spectroscopy techniques, and so contribute to addressing the long-standing question of the molecular mechanisms behind virus inactivation at liquid/solid interfaces.

\section{Performance of membranes on non-enveloped viruses}

When tested on non-enveloped viruses, the BLG AF-Fe membranes again showed an outstanding performance. An elimination efficiency of more than six orders of magnitude was found for MS2 (Fig. 4a), a non-enveloped bacteriophage of $\sim 28 \mathrm{~nm}$ diameter, which is very often used as a surrogate for human non-enveloped viruses $^{45}$. The infectious virus concentrations went from $\sim 10^{6}$ PFU ml ${ }^{-1}$ before filtration to below the detection limit after filtration. Neither the cellulose support nor BLG AF alone showed any detectable effect on the infectivity of filtered MS2, which again demonstrates the unique synergistic effect of the BLG AF-Fe membranes. By quantifying the genome count before and after filtration (Supplementary Fig. 10), we observed that most of the viruses were retained on the membrane material, but still with a detectable amount of viral genomes that went through the filter. After filtration, the ratio of infective to total viruses (considered to be equivalent to the genome count) decreased by several orders of magnitude (Supplementary Fig. 10). The observed results again indicate that in the case of non-enveloped viruses, BLG AF-Fe hybrid membranes not only retain the virus but also probably inactivate it. Further assessment of the virucidal effect of the membrane was conducted by recovering the MS2 viruses retained on the membrane filter in a similar approach to that done for $\Phi 6$. The total genomes detected in the filtrate and recovered from the membrane accounted for $\sim 65 \%$ of the total filtered viruses. Although it can neither be excluded that non-infective viruses are preferentially recovered over infective viruses nor that the viruses might be partially inactivated in the recovery process, we still observed a clear decrease in the ratio of infective-to-total viruses from 0.03 for the filtered solution to 0.01 of the recovered viruses (Fig. 4 b). Finally, we tested the membrane against enterovirus 71 (EV71), a highly robust virus that is known to retain its infectivity in the digestive tract and is also highly resistant to acidic conditions. Figure $4 \mathrm{c}$ shows that after filtration through BLG AF-Fe membranes the infectivity of EV71 went down to approximately one-third of the infectivity before filtration. No reduction in infectivity was observed for filtration through the cellulose support or the BLG AF alone, which demonstrates again the efficacy of the membranes developed.

\section{Sustainability footprint of the membranes}

To assess the performance, cost and environmental impact of the BLG AF-Fe membranes in a broader context, we evaluated its efficiency and sustainability footprint and benchmarked it against one of the most-used membrane technologies for virus removal: nanofiltration (NF). NF is a relatively simple process that retains a wide range of viruses via size exclusion, with removal efficiencies of 4-6 orders of magnitude ${ }^{46}$. The evaluation is based on the three pillars of sustainability, that is, techno-economic, environmental and social, reflected by eight discriminants: operating costs, investment cost, energy consumed, water recovery, removal efficiency, pressure, public acceptability and environmental friendliness. The performance of the technology in each indicator is ranked by either a low $(i=1)$, medium $(i=2)$ or high $(i=3)$ level score ${ }^{47}$. By doing so, semi-qualitative factors, such as public acceptability and environmental friendliness, can also be included in the assessment. 
As shown in Fig. 5, NF scores medium and low in most discriminants, due to its high price, pressure, energy consumption ${ }^{48}$ and the release of toxic organic solvents during fabrication ${ }^{49}$, which is a typical problem of polymeric membranes ${ }^{50}$. The overall sustainability footprint is estimated by summing up the individual components as $100 \% \times \sum_{j=1}^{8}\left(\frac{i}{3}\right)_{j} \times \frac{1}{8}$, where each of the $j=8$ factors carries a weight between $1 / 3$ and 1 depending on the score $i$. The sustainability footprints for BLG AF-Fe membranes and NF were estimated to 96 and 58\%, respectively, which clearly highlights the superiority of BLG AF-Fe membranes over conventional membrane processes for virus removal in terms of efficiency, cost and sustainability. Although the sustainability footprint of traditional membrane technologies can be slightly increased by considering less environmentally aggressive methods than NF, such as ultrafiltration for which the sustainability footprint is $\sim 63 \%$ (Supplementary Fig. 11), this comes at the expenses of virus removal efficiency, which drops down to insufficient values for safety requirements (for example, 2-4 orders of magnitude in ultrafiltration; Supplementary Fig. 11). Finally, we also showed that the mechanical stability of BLG AFFe can be further enhanced by introducing cellulose and carbon, which extends the time of service without a loss of performance (Supplementary Fig. 12).

\section{Concluding remarks}

In summary, we have shown the general and broad efficacy of AFFe membranes against both enveloped and non-enveloped viruses, which include key viruses such as SARS-CoV-2, H1N1 and EV71. The membrane introduced in this work is made by combining two widely available, food-grade components: AFs obtained by fibrillization of the milk protein BLG on which iron oxyhydroxide NPs are synthesized in situ from iron salts by simple $\mathrm{pH}$ changes, in a straightforward fabrication procedure. This is an antiviral filtration membrane made entirely by biosourced and biodegradable components. When combined with the outstanding virucidal properties of the membrane and the inactivation of the virus within it, these characteristics may allow a disposal of used membranes that is safe for both humans and the environment. Taken together, these results make this technology of immediate importance to mitigate current and future viral pandemics, as well as to address worldwide clean water challenges associated with pathogens.

\section{Online content}

Any methods, additional references, Nature Research reporting summaries, source data, extended data, supplementary information, acknowledgements, peer review information; details of author contributions and competing interests; and statements of data and code availability are available at https://doi.org/10.1038/ s41565-021-00920-5.

Received: 22 January 2021; Accepted: 27 April 2021; Published online: 3 June 2021

\section{References}

1. Liu, C. et al. Research and development on therapeutic agents and vaccines for COVID-19 and related human coronavirus diseases. ACS Cent. Sci. 6, 315-331 (2020).

2. Gall, A. M., Mariñas, B. J., Lu, Y. \& Shisler, J. L. Waterborne viruses: a barrier to safe drinking water. PLoS Pathog. 11, 1-7 (2015).

3. Reynolds, K. A., Mena, K. D. \& Gerba, C. Risk of waterborne illness via drinking water in the United States. Rev. Environ. Contam. Toxicol. 192 117-158 (2008).

4. Sinclair, R. G., Jones, E. L. \& Gerba, C. P. Viruses in recreational water-borne disease outbreaks: a review. J. Appl. Microbiol. 107, 1769-1780 (2009).

5. Sorrell, E. M., Wan, H., Araya, Y., Song, H. \& Perez, D. R. Minimal molecular constraints for respiratory droplet transmission of an avian-human H9N2 influenza A virus. Proc. Natl Acad. Sci. USA 106, 7565-7570 (2009).
6. Morawska, L. \& Cao, J. Airborne transmission of SARS-CoV-2: the world should face the reality. Environ. Int. 139, 105730 (2020).

7. Leung, N. H. L. et al. Respiratory virus shedding in exhaled breath and efficacy of face masks. Nat. Med. 26, 676-680 (2020).

8. Prather, K. A. et al. Airborne transmission of SARS-CoV-2. Science 370, 303-305 (2020)

9. Tellier, R. Review of aerosol transmission of influenza A virus. Emerg. Infect. Dis. 12, 1657-1662 (2006).

10. Patrício Silva, A. L. et al. Increased plastic pollution due to COVID-19 pandemic: challenges and recommendations. Chem. Eng. J. 405, 126683 (2021).

11. Ammendolia, J., Saturno, J., Brooks, A. L., Jacobs, S. \& Jambeck, J. R. An emerging source of plastic pollution: environmental presence of plastic personal protective equipment (PPE) debris related to COVID-19 in a metropolitan city. Environ. Pollut. 269, 116160 (2021).

12. Prata, J. C., Silva, A. L. P., Walker, T. R., Duarte, A. C. \& Rocha-Santos, T COVID-19 pandemic repercussions on the use and management of plastics. Environ. Sci. Technol. 54, 7760-7765 (2020).

13. Krammer, F. SARS-CoV-2 vaccines in development. Nature 586, 516-527 (2020).

14. Ball, $\mathrm{P}$. The lightning-fast quest for COVID vaccines-and what it means for other diseases. Nature 589, 16-18 (2021).

15. Khamsi, R. Can the world make enough coronavirus vaccine? Nature $\mathbf{5 8 0}$, 578-580 (2020)

16. Korber, B. et al. Tracking changes in SARS-CoV-2 spike: evidence that D614G increases infectivity of the COVID-19 virus. Cell 182, 812-827 (2020).

17. Weisblum, Y. et al. Escape from neutralizing antibodies by SARS-CoV-2 spike protein variants. eLife 9, e61312 (2020).

18. Boone, S. A. \& Gerba, C. P. Significance of fomites in the spread of respiratory and enteric viral disease. Appl. Environ. Microbiol. 73, 1687-1696 (2007).

19. Tuladhar, E. et al. Reducing viral contamination from finger pads: handwashing is more effective than alcohol-based hand disinfectants. J. Hosp. Infect. 90, 226-234 (2015).

20. Coia, J. E. et al. Guidance on the use of respiratory and facial protection equipment. J. Hosp. Infect. 85, 170-182 (2013).

21. Sangkham, S. Face mask and medical waste disposal during the novel COVID-19 pandemic in Asia. Case Stud. Chem. Environ. Eng. 2, 100052 (2020).

22. Tran, H. N. et al. SARS-CoV-2 coronavirus in water and wastewater: a critical review about presence and concern. Environ. Res. 193, 110265 (2021).

23. Drinking-water: Key Facts (World Health Organization, 2019); https://www. who.int/news-room/fact-sheets/detail/drinking-water

24. Ramani, S. \& Kang, G. Viruses causing childhood diarrhoea in the developing world. Curr. Opin. Infect. Dis. 22, 477-482 (2009).

25. Prevost, B. et al. Viral persistence in surface and drinking water: suitability of PCR pre-treatment with intercalating dyes. Water Res. 91, 68-76 (2016).

26. Carratalà, A., Bachmann, V., Julian, T. R. \& Kohn, T. Adaptation of human enterovirus to warm environments leads to resistance against chlorine disinfection. Environ. Sci. Technol. 54, 11292-11300 (2020).

27. Sigstam, T. et al. Subtle differences in virus composition affect disinfection kinetics and mechanisms. Appl. Environ. Microbiol. 79, 3455-3467 (2013).

28. Wigginton, K. R., Ye, Y. \& Ellenberg, R. M. Emerging investigators series: the source and fate of pandemic viruses in the urban water cycle. Environ. Sci. Water Res. Technol. 1, 735-746 (2015).

29. Bogler, A. et al. Rethinking wastewater risks and monitoring in light of the COVID-19 pandemic. Nat. Sustain. 3, 981-990 (2020)

30. Bivins, A. et al. Persistence of SARS-CoV-2 in water and wastewater. Environ Sci. Technol. Lett. 7, 937-942 (2020).

31. Hornstra, L. M. et al. Monitoring the integrity of reverse osmosis membranes using novel indigenous freshwater viruses and bacteriophages. Environ. Sci. Water Res. Technol. 5, 1535-1544 (2019).

32. Ale, A. et al. Exposure to a nanosilver-enabled consumer product results in similar accumulation and toxicity of silver nanoparticles in the marine mussel Mytilus galloprovincialis. Aquat. Toxicol. 211, 46-56 (2019).

33. Abramenko, N. et al. The effect of capping agents on the toxicity of silver nanoparticles to Danio rerio embryos. Nanotoxicology 13, 1-13 (2019).

34. Aquino De Carvalho, N., Stachler, E. N., Cimabue, N. \& Bibby, K. Evaluation of Phi6 persistence and suitability as an enveloped virus surrogate. Environ. Sci. Technol. 51, 8692-8700 (2017).

35. Farley, M. M. 2009 H1N1 influenza: a twenty-first century pandemic with roots in the early twentieth century. Am. J. Med. Sci. 340, 202-208 (2010).

36. Pecson, B. M., Decrey, L. \& Kohn, T. Photoinactivation of virus on iron-oxide coated sand: enhancing inactivation in sunlit waters. Water Res. 46, $1763-1770$ (2012).

37. Block, K. A. et al. Disassembly of the cystovirus $\Phi 6$ envelope by montmorillonite clay. MicrobiologyOpen 3, 42-51 (2014). 
38. Kosmulski, M. Isoelectric points and points of zero charge of metal (hydr) oxides: 50 years after Parks' review. Adv. Colloid Interface Sci. 238, $1-61$ (2016).

39. Michen, B. \& Graule, T. Isoelectric points of viruses. J. Appl. Microbiol. 109, 388-397 (2010).

40. Nieto-Juarez, J. I. \& Kohn, T. Virus removal and inactivation by iron (hydr) oxide-mediated Fenton-like processes under sunlight and in the dark. Photochem. Photobiol. Sci. 12, 1596-1605 (2013).

41. Ryan, J. N. et al. Field and laboratory investigations of inactivation of viruses (PRD1 and MS2) attached to iron oxide-coated quartz sand. Environ. Sci. Technol. 36, 2403-2413 (2002).

42. Harvey, R. W. \& Ryan, J. N. Use of PRD1 bacteriophage in groundwater viral transport, inactivation, and attachment studies. FEMS Microbiol. Ecol. 49, 3-16 (2004).

43. Vajda, J. et al. Mono- and polyprotic buffer systems in anion exchange chromatography of influenza virus particles. J. Chromatogr. A 1448 , 73-80 (2016).

44. Pandey, L. M. Surface engineering of personal protective equipments (PPEs) to prevent the contagious infections of SARS-CoV-2. Surf. Eng. 36, 901-907 (2020).

45. Shirasaki, N., Matsushita, T., Matsui, Y., Marubayashi, T. \& Murai, K. Investigation of enteric adenovirus and poliovirus removal by coagulation processes and suitability of bacteriophages MS2 and $\varphi$ X174 as surrogates for those viruses. Sci. Total Environ. 563-564, 29-39 (2016).
46. Shokri Doodeji, M. \& Zerafat, M. A review on the applications of nanofiltration in virus removal and pharmaceutical industries. Glob. J. Nanomed. 3, 1-3 (2018).

47. Bolisetty, S., Peydayesh, M. \& Mezzenga, R. Sustainable technologies for water purification from heavy metals: review and analysis. Chem. Soc. Rev. 48, 463-487 (2019)

48. Muga, H. E. \& Mihelcic, J. R. Sustainability of wastewater treatment technologies. J. Environ. Manag. 88, 437-447 (2008).

49. Razali, M. et al. Sustainable wastewater treatment and recycling in membrane manufacturing. Green Chem. 17, 5196-5205 (2015).

50. Szekely, G., Jimenez-Solomon, M. F., Marchetti, P., Kim, J. F. \& Livingston, A. G. Sustainability assessment of organic solvent nanofiltration: from fabrication to application. Green Chem. 16, 4440-4473 (2014).

51. ViralZone (Swiss Institute of Bioinformatics); www.expasy.org/viralzone

52. Kurpiewska, K. et al. Investigation of high pressure effect on the structure and adsorption of $\beta$-lactoglobulin. Colloids Surf. B 161, 387-393 (2018).

53. Berman, H. M. et al. The protein data bank. Acta Crystallogr. D 58, 899-907 (2002).

Publisher's note Springer Nature remains neutral with regard to jurisdictional claims in published maps and institutional affiliations.

(C) The Author(s), under exclusive licence to Springer Nature Limited 2021 


\section{Methods}

A full detailed description of the materials and methods used in this work is provided in the Supplementary Information. A brief summary is given below.

Materials. The protein BLG was purified from whey protein isolate received as a kind gift from Fonterra.

For the viruses, $\Phi 6$ bacteriophage (21518) and MS2 bacteriophage (13767) were from the DSMZ culture collection; SARS-CoV-2/Switzerland/GE9586/2020 was isolated from a clinical specimen in the University Hospital in Geneva and replicated twice in Vero-E6 before the experiments. SARS-CoV-2/human/ Switzerland/IMV5/2020 was isolated from a clinical specimen at the Institute of Medical Virology, University of Zurich and has been described previously ${ }^{54,55}$.

Human H1N1 virus A/Netherlands/602/2009 was a gift from M. Schmolke (Department of Microbiology and Molecular Medicine). EV71 was isolated from a clinical specimen at the University Hospital of Geneva in rhabdomyosarcoma cells. Cells were infected and the supernatant was collected 2 days postinfection, clarified, aliquoted and frozen at $-80^{\circ} \mathrm{C}$ before titration by plaque assay in rhabdomyosarcoma cells.

Methods. Purification of BLG monomers and preparation of AFs. The purification of BLG from the whey protein and the preparation of AFs from the purified protein are discussed in a previous report ${ }^{56}$.

Preparation of BLG AF-Fe hybrids. AFs coated with Fe NPs were obtained by mixing AF solution at $\mathrm{pH} 2$ with an aqueous solution of $\mathrm{FeCl}_{3} \cdot 6 \mathrm{H}_{2} \mathrm{O}$ and adjusting the $\mathrm{pH}$ to 7 with $\mathrm{NaOH}$.

Preparation of BLG monomer-Fe NPs. BLG monomer-Fe NPs were prepared using a similar protocol as that used to prepare the BLG AF-Fe hybrids, but using BLG monomers instead of BLG AF.

Preparation of BLG AF-Fe membranes. A syringe-aided filtration set-up was used to prepare BLG AF-Fe membranes. A cellulose support with a $0.45 \mu \mathrm{m}$ pore size was inserted into the filtration set-up and placed on a glass bottle. BLG AF-Fe hybrids $(8 \mathrm{ml})$ were taken with the help of a syringe and injected into the filtration system, and extra water was drained to form a membrane.

Characterization of BLG AF-Fe hybrids by XPS. XPS was used to determine the $\mathrm{AF}-\mathrm{Fe}$ hybrid composition. Iron(II) and iron(III) hydroxides were found to contribute to 26.4 and $63.2 \%$ of the total peak area, respectively, along with $10.4 \%$ of iron chloride (iron(II) + iron(III) chloride).

Additional methods used. Experimental details on inactivation of the $\Phi 6$, MS2, EV71, SARS-CoV-2 and $\mathrm{H} 1 \mathrm{~N} 1$ viruses via the incubation of different concentrations of BLG AF-Fe hybrids, filtration experiments, viral genome extraction and real-time reverse transcription polymerase chain reaction (RT-qPCR) are given in full in the Supplementary Information along with cytotoxicity tests on MDCK cells.

Details on cryo-SEM, SEM, TEM and cryo-TEM are also given in full in the Supplementary Information.

\section{Data availability}

All the data generated and analysed during this study are included in the article and its Supplementary Information. Source data are provided with this paper.

\section{References}

54. Busnadiego, I. et al. Antiviral activity of type I, II, and III interferons counterbalances ACE2 inducibility and restricts SARS-CoV-2. MBio 11, $1-10$ (2020).

55. Pohl, M. O. et al. SARS-CoV-2 variants reveal features critical for replication in primary human cells. PLoS Biol. 19, e3001006 (2021).

56. Jung, J. M., Savin, G., Pouzot, M., Schmitt, C. \& Mezzenga, R. Structure of heat-induced $\beta$-lactoglobulin aggregates and their complexes with sodium-dodecyl sulfate. Biomacromolecules $\mathbf{9}$, 2477-2486 (2008).

\section{Acknowledgements}

We acknowledge support by the Innosuisse CTI grant no. 27165.1 PFLS-LS and the Swiss National Science Foundation project no. 31CA30_196217. W. Gruissem and M. Loessner at ETHZ are gratefully acknowledged for allowing access to their laboratory facilities, and E. Moritz (University of Zurich) for providing technical support. We acknowledge the support of the Scientific Center for Optical and Electron Microscopy (ScopeM) of ETH Zurich. M.G. acknowledges the support of NCCR Bio-Inspired Materials.

\section{Author contributions}

A.P. carried out the experiments that involved the $\Phi 6$ bacteriophage and MS2 bacteriophage. A. Rahimi and S.B. carried out the synthesis of AF-Fe NPs. S.H. carried out the cryo-SEM, SEM, TEM and cryo-TEM experiments. C.M. carried out the experiments that involved H1N1, enteroviruses and RT-qPCR of SARS-CoV-2 under the supervision of C.T. M.G. carried out the filtration experiments of SARS-CoV-2 under the supervision of F.S. M.O.P. and I.B. carried out the SARS-CoV-2 incubation experiments under the supervision of B.G.H. and S.S. C.G. propagated and purified $\Phi 6$ and MS2 for electron microscopy imaging and RT-qPCR. A.A. designed the experiments on the determination of the inactivation mechanism for all viruses, RT-qPCR experiments for $\Phi 6$ and MS2, and propagation protocols for $\Phi 6$ and MS2; analysed the data and compiled the figures. A. Rossi carried out, processed and interpreted the XPS experiments. R.B.A. designed and conducted the RT-qPCR for $\Phi 6$ and MS2. M.P. and R.M. performed the evaluation of the sustainability footprint of the BLG AF-Fe and NF technologies. R.M. designed and directed the study, and analysed the data. A.P., A.A. and R.M. wrote the manuscript. All the authors edited and approved the final manuscript.

\section{Competing interests}

R.M., A.P., S.B. and A. Rahimi are the inventors of a filed patent application related to the work presented here. All the other authors declare no competing interests.

\section{Additional information}

Supplementary information The online version contains supplementary material available at https://doi.org/10.1038/s41565-021-00920-5.

Correspondence and requests for materials should be addressed to R.M.

Peer review information Nature Nanotechnology thanks Volodymyr Tarabara and T. Waite for their contribution to the peer review of this work.

Reprints and permissions information is available at www.nature.com/reprints. 